\title{
Direct acting anti-hepatitis C combinations as potential COVID- 19 protease inhibitors
}

\author{
Mahmoud Hamed $^{1} \cdot$ Mahmoud El-Hasab $^{2} \cdot$ Fotouh R. Mansour $^{1,3}$ (D
}

Received: 14 January 2021/Accepted: 17 April 2021/Published online: 29 April 2021

(C) Indian Virological Society 2021

\begin{abstract}
The coronavirus pandemic could be the most threatening outbreak in the twenty-first century. According to the latest records of world health organization, more than 130 millions have been infected by COVID-19, with more than 2.9 million reported deaths. Yet, there is no magic cure for treatment of COVID-19. The concept of drug repurposing has been introduced as a fast, life-saving approach for drug discovery. Drug repurposing infers investigating already approved drugs for new indications, using the available information about pathophysiology of diseases and pharmacodynamics of drugs. In a recent work, more than 3000 FDA approved drugs were tested using virtual screening as potential antiviral agents for COVID19. In this work, the top ranked five hits from the previous docking results together with drugs of similar chemical feature and/or mechanistic destinations were further tested using AutoDock Vina. The results showed that anti-HCV combinations could be potential therapeutic regimens for COVID-19 infections.
\end{abstract}

Supplementary information The online version contains supplementary material available at https://doi.org/10.1007/s13337021-00691-6.

Fotouh R. Mansour

fotouhrashed@pharm.tanta.edu.eg

1 Pharmaceutical Services Center, Faculty of Pharmacy, Tanta University, Tanta 31111, Egypt

2 Department of Pharmaceutical Chemistry, Faculty of Pharmacy, Badr University in Cairo (BUC), Cairo, Egypt

3 Pharmaceutical Analytical Chemistry Department, Faculty of Pharmacy, Tanta University, El-Geish Street, The Medical Campus, Tanta 31111, Egypt
Keywords Coronavirus pandemic · COVID-19 . AutoDock Vina - Anti-hepatitis C combinations · Direct acting antiviral agent

\section{Introduction}

The coronavirus pandemic is a global risk that threatens the lives of millions of people on earth. This pandemic is caused by severe acute respiratory syndrome-coronavirus 2 (SARS-CoV-2) (also known as coronavirus disease 2019; COVID-19) [1], which was first diagnosed in Wuhan, the capital of china's Hubei province and spread since then to the rest of the world. According to Weekly epidemiological update of the world health organization, more than 130 million reported cases of coronavirus infections were reported worldwide, with number of deaths exceeding 2.9 million. Despite the increasing numbers of cases and deaths, there is no magic cure for SARS-CoV-2 yet. The process of drug discovery takes years of extensive research, and requires millions of dollars to introduce a single drug to the pharmaceutical market [2]. The first stage in the process of drug discovery starts with laboratory testing, which takes in average 3-4 years. Successful molecules in the first stage are tested on experimental animals, a stage that takes around 3 years. Drugs with proven safety and efficacy will be further tested on human through three phases: Phase I (tens of healthy volunteers), Phase II (hundreds of patients), and Phase III (thousands of patients). The real challenge in the drug discovery journey, besides the prolonged timeframe and the high cost, is the low success rate; out of 5000 molecules that undergo laboratory testing, only 250 enter the preclinical stage on experimental animals. Out of these 250 molecules, only 5 molecules are transferred to the clinical trial stage to finally 
launch a single drug. Figure 1 shows the different stages of drug discovery and the estimated timeframe.

The concept of drug repurposing has been recently presented as a shortcut to the expensive long process of drug discovery. Drug repurposing implies investigating already approved drugs for new indications, using the available information about pathophysiology of diseases and pharmacodynamics of drugs. SARS-CoV-2 is a positive RNA strand that shares about $79.6 \%$ genome identity with SARS-CoV and about $96 \%$ with bat coronavirus [3]. This similarity between SARS-CoV-2 and other coronaviruses can hypothesize how the host immune system may react to this particular virus as well as the virus response [4]. During SARS-CoV viral infection, the trimeric spike glycoprotein is cleaved into two subunits: S1 and S2. The $\mathrm{S} 1$ subunit binds to the peptidase domain (PD) of the angiotensin converting enzyme 2 (ACE2) through the receptor binding domain (RBD) [5, 6]. Another critical process for viral infection is the cleavage of $\mathrm{S} 2$ by the host protease enzyme [7], which makes this enzyme an attractive target for researchers. Figure 2 shows the life cycle of SARS-CoV-2 and the potential targets for drug development.

In a recent study by Alessandro Contini, more than 3000 FDA approved drugs were tested using virtual screening by PLANTS software for potential use as antiviral agents for SARS-CoV-2. The results showed that a number of the known protease inhibitors could be successful candidates of COVID-19 therapy [8]. As an extension of Contini's work, the top ranked five hits from the docking results [8] were selected for further screening by AutoDock Vina. To widen the scope of our screening, drugs with similar chemical feature and/or mechanistic destinations to the top five hits were also tested and compared with the reported promising drugs of COVID-19, based on the calculated

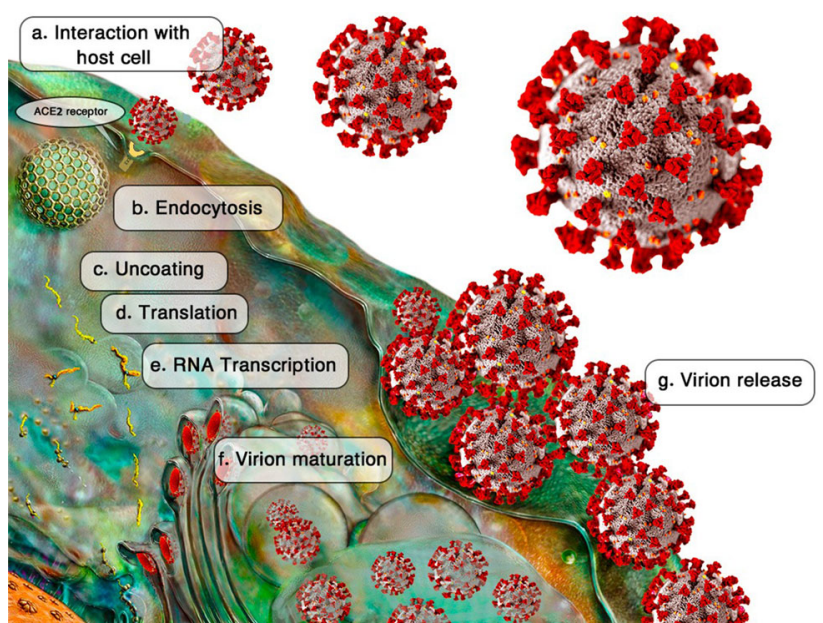

Fig. 2 The life cycle of SARS-CoV-2 showing the possible target site

binding affinity. AutoDock is the most commonly cited docking program in the scientific literature [9].

\section{Materials and method}

\section{Protein/macromolecule/receptor}

COVID-19 main protease (PDB ID: 6M03) was obtained from the Protein Data Bank [10] in a pdb format with a resolution of $2 \AA$. The pdb file COVID-19 main protease was a textual format describing the 3D structure of the enzyme. The 6M03 protein was presented in apo form (no ligand bound) and contained one chain (chain A), with a sequence length of 306 amino acids.
Fig. 1 Overview of drug discovery process showing the timeframe of each stage

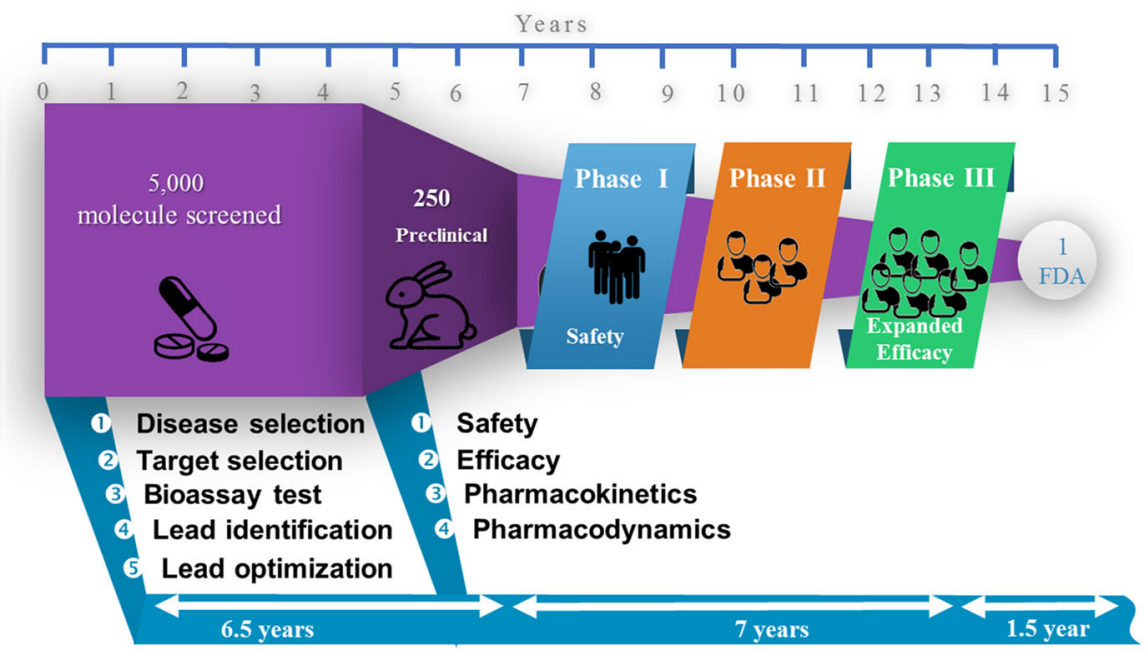




\section{Ligands}

The top five hits (Angiotensin II human acetate, indinavir, GHRP-2, cabicistat and montelukast), the structurally similar drugs and the other proteases inhibitors were tested. Similar compounds of the top five hits were selected using the Drug Bank with a similarity threshold of 0.7. For sake of comparison, the binding affinity of promising drugs were also calculated. Figure 3 shows the tested drugs and the reason of testing. Drug-like properties were calculated for experimental drugs using "rule of five" which predicts the absorption and the permeation (Table S1). The SMILES (simplified molecular input line entry system) strings of the selected compounds were collected from PubChem database (pubchem.ncbi.nlm.nih.gov) and Drug Bank database (https://databank.worldbank.org), then submitted to UCSF Chimera to generate 3D Structures and for energy minimization.

\section{Molecular docking}

The 3D model of COVID-19 protease and the ligands were then prepared in the right format (pdbqt) using Molecular Graphics Laboratory (MGL) tools for conducting docking using Auto Dock Vina [11]. MGL 1.5.6 tools were used to generate grid for the receptor. Discovery Studio Visualizer was used to visualize the results of docking, and the evaluation was based on the binding affinity.

\section{Result and discussion}

Virtual screening of the selected collection of ligands showed marked variations in the Dock score. In this section, only drugs with dock scores higher than the top drugs in Contini's work will be highlighted. The following table shows tested drugs in a descending order according to the calculated dock score.

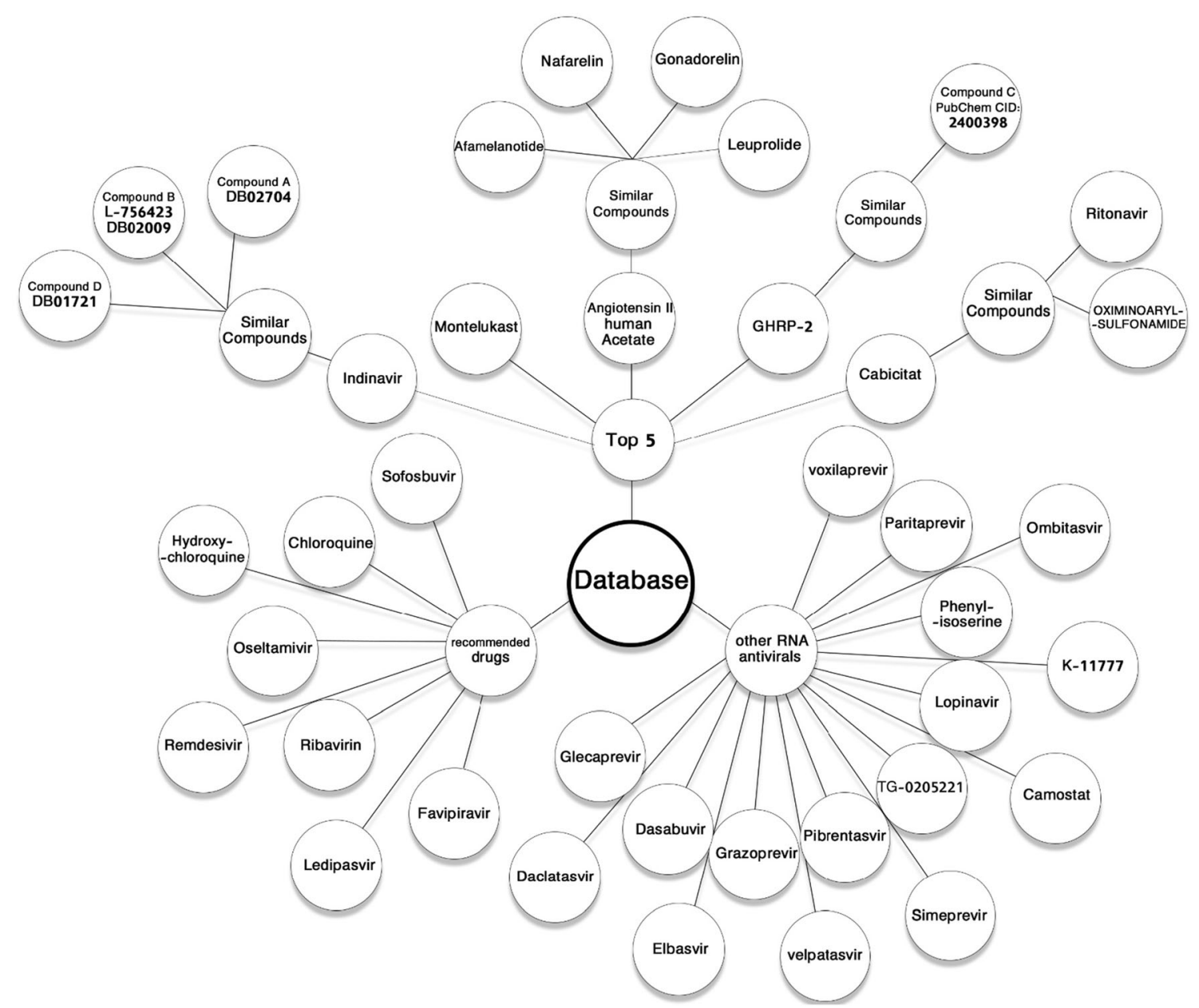

Fig. 3 Data base used for screening for potential COVID-19 protease inhibitor including Contini's top five hits, similar chemical feature and/or mechanistic destinations to the top five hits and compared the reported promising drugs COVID-19 


\section{Drugs with binding affinity higher than ivermectin}

As shown in Table 1, three of the tested drugs (Simeprevir, Ledipasvir and pibrentasvir) showed binding affinity higher than ivermectin. Interestingly, the three drugs are antiviral for hepatitis $\mathrm{C}$ infection with different modes of action. Simeprevir is a hepatitis C virus (HCV) NS3/4A protease inhibitor indicated for the treatment of chronic hepatitis $\mathrm{C}$ virus (HCV) infection in patients with $\mathrm{HCV}$ genotype 1 or 4 [12]. Simeprevir was approved by the FDA in November 2014 and is marketed under the brand name Olysio ${ }^{\circledR}$ as oral tablets. Ledipasvir is a direct acting antiviral (DAA) and prescribed in combinations to treat chronic HCV genotypes $1,4,5$, and 6 . Although ledipasvir's mechanism of action is unknown, it is postulated to prevent hyperphosphorylation of NS5A (Non-structural protein 5A), which is required for viral protein reproduction, RNA replication and assembly of HCV virions [13]. Pibrentasvir inhibits HCV NS5A; a protein that is essential for replication, assembly and maturation of infectious viral proteins. Pibrentasvir is indicated for patients with chronic hepatitis $\mathrm{C}$ virus (HCV) genotype 1, 2, 3, 4, 5 or 6 infection with or without compensated cirrhosis [14]. Ivermectin was first used against worms (except tapeworms), but, in 2012 it was approved for the treatment of head lice infestations in patients 6 months of age [15]. Ivermectin is mainly used for the treatment of intestinal strongyloidiasis resulting from Strongyloides stercoralis and onchocerciasis resulting from Onchocerca volvulus and scabies caused by an infestation of the skin by Sarcoptes scabiei [16]. Ivermectin showed ability to induce $\sim 5000$-fold reduction in SARS-CoV-2 RNA within two days [17]. The mechanism of action of ivermectin on in SARS-CoV-2 RNA is not confirmed, but the calculated binding affinity speculates the possibility of competitive binding to viral protease [17].

\section{Drugs with binding affinity higher than the top five hits}

As shown in Table 1, seven drugs showed binding affinities higher than the five top hits, but less than ivermectin. These drugs included compound (A), glecaprevir, paritaprevir, elbasvir, daclatasvir, velpatasvir and compound (B). Compound (A) -(2R,3R,4R,5R)-3,4-Dihydroxy-N,N'bis[(1S,2R)-2-hydroxy-2,3-dihydro-1H-inden-1-yl]-2,5bis(2-phenylethyl)hexanediamide- showed high binding affinity to COVID-19 protease. This ligand is not approved yet as an FDA drug, and it is a synonym for diol-based HIV-1 protease inhibitor 4. The compound computed properties are compared to the properties typically used for druglikeness and leadlikeness (Table S1) according to the "rule of five", which helps optimize pharmacokinetic properties of drug molecules. Glecaprevir is a direct acting antiviral agent and HCV NS3/4A protease inhibitor, and is indicated for adult patients with chronic HCV genotype 1, $2,3,4,5$ or 6 with compensated cirrhosis or infection without cirrhosis [18]. Paritaprevir is used in combination with other drugs for treatment of HCV genotype $1 \mathrm{a}, 1 \mathrm{~b}$ and
Table 1 Binding affinity score with COVID-19 protease (6M03)

\begin{tabular}{llll}
\hline Drug name & Dock score & Drug name & Dock score \\
\hline Simeprevir & -9.5 & Afamelanotide & -7.4 \\
Ledipasvir & -9.4 & Sofosbuvir & -7.3 \\
Pibrentasvir & -9.4 & Camostat & -7.1 \\
Ivermectin & -8.7 & Lopinavir & -7.1 \\
Compound (A) & -8.6 & Indinavir & -7.0 \\
Glecaprevir & -8.4 & Ritonavir & -7.0 \\
Paritaprevir & -8.2 & Gonadorelin & -6.7 \\
Elbasvir & -8.2 & Leuprolide & -6.7 \\
Daclatasvir & -7.9 & Remdesivir & -6.5 \\
velpatasvir & -7.9 & TG-0205221 & -6.4 \\
Compound (B) & -7.9 & GHRP-2 & -6.4 \\
Angiotensin II human Acetate & -7.9 & Oximinoaryl sulfonamide & -6.2 \\
voxilaprevir & -7.8 & Cabicistat & -6.0 \\
K-11777 & -7.7 & Ribavirin & -5.6 \\
Grazoprevir & -7.6 & Chloroquine & -5.5 \\
Dasabuvir & -7.5 & Oseltamivir & -5.3 \\
Compound (C) & -7.5 & Phenylisoserine & -5.2 \\
Compound (D) & -7.5 & Favipiravir & -5.1 \\
Ombitasvir & -7.4 & Montelukast & -4.6 \\
& & Hydroxychloroquine & -4.4 \\
\hline & & &
\end{tabular}


4 by inhibition the of NS3/4A serine protease [19]. Elbasvir is part of combination therapy for treatment of chronic Hepatitis C. It is a direct acting antiviral by targeting HCV non-structural protein $5 \mathrm{~A}$ with inhibition effect, the role of the protein is unknown but it is obvious that the protein is essential for viral replication and virion assembly [20]. Angiotensin II human acetate showed the highest binding affinity in Contini's work. The drug is authorized for use in the European Union (Giapreza) and indicated for the treatment of refractory hypotension in adults with sepsis [21]. The compound has interacted with the target with a binding affinity score of $-7.9(\mathrm{kcal} / \mathrm{mol})$, but Angiotensin II human acetate is a natural substrate for protease. It is here worth mentioning that three drugs (Velpatasvir, daclatasvir and compound B) showed binding capacity equivalent to angiotensin II human acetate. Daclatasvir is a direct-acting antiviral agent used for the treatment of $\mathrm{HCV}$ genotype 1 and 3. The target of daclatsvir is Nonstructural protein 5A [22]. Velpatasvir is an HCV NS5A inhibitor used in combination with other drugs for the treatment of patients with chronic $\mathrm{HCV}$ genotypes 1, 2, 3, 4, 5 or 6 infection without cirrhosis or with compensated cirrhosis, or in combination with ribavirin if associated with decompensated cirrhosis [23]. Compound B (L-756423) is still experimental, and completed phase 2 in treatment of human immunodeficiency virus (HIV) Infections by targeting gag-pol polyprotein [24]. The ligand protein interaction score was $-7.9(\mathrm{kcal} / \mathrm{mol})$. So, the ligand might have the ability to inhibit Covid-19 protease. Figure S1 shows the binding sites of the drugs with highest dock scores in this study.

\section{Other potential protease inhibitors}

As Table 1 shows, the other tested drugs exhibited lower dock scores than angiotensin II human acetate. In this section, the indication and the mode of action of the highest 14 these drugs are briefly discussed. K-11777 is a cysteine protease inhibitor that acts as a broad-spectrum antiviral, by targeting cathepsin-mediated cell entry. K-11777 was observed to inhibit SARS-CoV virus entry in the subnanomolar range. So, potential inhibitory effect of $\mathrm{K}-11777$ on COVID-19 protease is possible. Grazoprevir is used for treatment of chronic hepatitis $\mathrm{C}$ in combination with other drugs as a direct acting antiviral medication. The drug is used for genotypes $1 \mathrm{a}, 1 \mathrm{~b}$, or 4 infection in adults [23], by the inhibition of NS3/4a protease. Which leads to the inhibition of $\mathrm{HCV}$ viral replication [25]. Dasabuvir is indicated for the treatment of patients with HCV genotype la or genotype $1 \mathrm{~b}$ in combination with other drugs, including those with compensated cirrhosis. Dasabuvir Inhibits HCV RNA-dependent RNA polymerase, the enzyme is encoded by the NS5B gene, and is essential for the replication of viral genome [26].

Compound (C) (2S)-N-Benzyl-2-acetamido-3-(1H-indol-3-yl)propanamide interacted with the target with binding affinity of -7.5 , but still not approved as therapeutic drug. The compound has good computed properties not only for drug likeness criteria but also for leadlikeness criteria in accordance with the "rule of five" (Table S1). Compound (D) (N-[2-hydroxy-1-indanyl]-5-[(2-tertiarybutylaminocarbonyl)-4(benzo[1,3]dioxol-5-ylmethyl)piperazino]-4-hydroxy-2-(1-phenylethyl)-pentanamide) is similar compound to indinavir with a similarity score of 0.789 and it is targeting gag-pol polyprotein [27], but the action of the compound is not available. The ligand interacted with the target with a score of $-7.5 \mathrm{kcal} / \mathrm{mol}$.

Ombitasvir is a direct acting antiviral used for the treatment of patients with genotype 4 chronic HCV infection without cirrhosis, by the inhibition of $\mathrm{HCV}$ nonstructural protein $5 \mathrm{~A}$, which required for viral replication, but the main role is not clear yet. Afamelanotide is currently the only approved drug therapy used in the management of erythropoietic protoporphyria, and has greater affinity for its target and a longer biological half-life [26]. The drug interacted with the target with a score of $7.4 \mathrm{kcal} / \mathrm{mol}$. Despite afamelanotide is peptide analogue of the endogenous alpha melanocyte-stimulating hormone $(\alpha-$ $\mathrm{MSH}$ ), the drug is more resistant to degradation by serum and proteolytic enzymes [28], so it is expected to resist degradation by COVID-19 protease. Sofosbuvir (Sovaldi $\left.{ }^{\circledR}\right)$ is a direct anti-viral used to treat the infectious liver disease caused by HCV by preventing viral replication through binding to the two $\mathrm{Mg}^{2+}$ ions present in $\mathrm{HCV}$ NS5B polymerase's GDD active site motif. The drug is used in combination with other drugs [29]. Sofosbuvir showed a binding affinity with a score of $-7.3 \mathrm{kcal} / \mathrm{mol}$ in the ligand protein interaction with COVID-19 protease. Camostat is a potent serine protease inhibitor, which is expected to have the ability to inhibit protease enzyme. Clinical trials on the drug has been performed with Estimated Enrollment of 180 participant [30]. Lopinavir isan antiviral, used in combination with other drugs in the treatment of HIV infection, and the drug is HIV protease inhibitor, which is responsible for the cleavage of gag polyprotein [31]. Lopinavir is now under study for repurposing for the treatment of COVID-19 in combination with ritonavir umifenovir [32]. Indinavir is an antiretroviral drug for the treatment of HIV infection, by targeting HIV type 1 protease [33]. Indinavir approved for medical use in 1996 [34]. The drug docking score of the interaction between the drug and COVID-19 protease was - 7.0 (kcal/mol). Ritonavir is an HIV protease inhibitor, and indicated in combination with other antiretroviral agents for the treatment of HIV-1 infection [35]. Ritonavir was first approved 
on March, 1996 by FDA [36]. The drug targets are nuclear receptor subfamily 1 group I member 2 and HIV type 1 protease. The action of ritonavir on both targets is activating and inhibiting respectively, so it is expected that the drug might inhibit COVID-19 protease as in ligand protein interaction with dock score $-7.0(\mathrm{kcal} / \mathrm{mol})$. Gonadorelin is a synthetic decapeptide gonadotropin-releasing hormone (GnRH). Gonadorelin is indicated for increasing the functional capacity and response of the gonadotropes of the anterior pituitary and after removal of a pituitary tumor by increasing residual gonadotropic function of the pituitary. Gonadorelin has agonist action on both gonadotropin-releasing hormone receptor and putative gonadotropin-releasing hormone II receptor [37], thus it might have inhibiting effect on COVID-19 protease as the score of ligand protein interaction with the target is -6.7 (kcal/mol).

\section{Hepatitis $\mathbf{C}$ combination drugs}

Hepatitis $\mathrm{C}$ infection is caused by hepatitis $\mathrm{C}$ virus which can deteriorate from chronic inflammation to fibrosis, cirrhosis and even to hepatocellular carcinoma [38]. Combination of $\mathrm{HCV}$ infection was recommended to increases treatment efficacy. These combinations include ledipasvir/sofosbuvir (Harvoni ${ }^{\circledR}$ ) dasabuvir/ombitasvir/paritaprevir/ritonavir (Viekira PAK $®$ ), elbasvir/grazoprevir (Zepatier $\left.{ }^{\circledR}\right), \quad$ ombitasvir/paritaprevir/ritonavir (Technivie ${ }^{\circledR}$ ) sofosbuvir/velpatasvir (Epclusa ${ }^{\circledR}$ ), glecaprevir/pibrentasvir (Mavyret $\left.{ }^{\circledR}\right)$. The pharmacokinetic and the pharmacodynamic profiles of these combinations have proven safety. Using these combinations as antivral agents for COVID-19 based on the results of the docking score could also guarantee synergistic effects without any unexpected drug-drug interactions. As shown in Table 1, Mavyret ${ }^{\circledR}$ combination is the most recommended. Harvoni ${ }^{\circledR}$ and Zepatier ${ }^{\circledR}$ combinations are also recommended due to the high dock score and the reported safety.

The current outbreak of coronavirus disease 2019 (COVID-19), caused by the severe acute respiratory syndrome coronavirus 2 (SARS-CoV-2) is still threatening a large number of people worldwide. This study aimed to examine several compounds that may be used to inhibit the disease. Antiviral drugs for $\mathrm{HCV}$ infection showed the highest binding affinity ( 13 drugs out of the top 20 drugs). The binding affinity of ivermectin is high compared with other drugs so, we the binding affinity of Ivermectin as a benchmark. Simeprevir, pibrentasvir and ledipasvir were the most promising candidates for COVID-19 protease but further research and investigation are required to confirm the efficiency of these compounds.

\section{References}

1. Rothan HA, Byrareddy SN. The epidemiology and pathogenesis of coronavirus disease (COVID-19) outbreak. J Autoimmun. 2020;109:102433.

2. Dickson M, Gagnon JP. Key factors in the rising cost of new drug discovery and development. Nat Rev Drug Discov. 2004;3:417-29.

3. Zhou P, Yang X-L, Wang X-G, Hu B, Zhang L, Zhang W, et al. A pneumonia outbreak associated with a new coronavirus of probable bat origin. Nature. 2020;579:270-3.

4. Prompetchara E, Ketloy C, Palaga T. Immune responses in COVID-19 and potential vaccines: lessons learned from SARS and MERS epidemic. Asian Pac $\mathrm{J}$ Allergy Immunol. 2020;38:1-9.

5. Song W, Gui M, Wang X, Xiang Y. Cryo-EM structure of the SARS coronavirus spike glycoprotein in complex with its host cell receptor ACE2. PLOS Pathog. 2018;14:e1007236.

6. Simmons G, Zmora P, Gierer S, Heurich A, Pöhlmann S. Proteolytic activation of the SARS-coronavirus spike protein: cutting enzymes at the cutting edge of antiviral research. Antivir Res. 2013;100:605-14.

7. Belouzard S, Chu VC, Whittaker GR. Activation of the SARS coronavirus spike protein via sequential proteolytic cleavage at two distinct sites. Proc Natl Acad Sci USA. 2009;106:5871-6.

8. Contini A. Virtual screening of an FDA approved drugs database on two COVID-19 coronavirus proteins. ChemRxiv; 2020.

9. Sousa SF, Fernandes PA, Ramos MJ. Protein-ligand docking: current status and future challenges. Proteins Struct Funct Genet. 2006;65:15-26.

10. Mittal L, Kumari A, Srivastava M, Singh M, Asthana S. Identification of potential molecules against COVID-19 main protease through structure-guided virtual screening approach. J Biomol Struct Dyn. 2020;2020:1-19.

11. Trott O, Olson AJ. AutoDock Vina: improving the speed and accuracy of docking with a new scoring function, efficient optimization, and multithreading. J Comput Chem. 2009;31:455-61.

12. Lawitz E, Matusow G, DeJesus E, Yoshida EM, Felizarta F, Ghalib R, et al. Simeprevir plus sofosbuvir in patients with chronic hepatitis $\mathrm{C}$ virus genotype 1 infection and cirrhosis: a phase 3 study (OPTIMIST-2). Hepatology. 2016;64:360-9.

13. Kwon HJ, Xing W, Chan K, Niedziela-Majka A, Brendza KM, Kirschberg T, et al. Direct Binding of Ledipasvir to HCV NS5A: mechanism of resistance to an HCV antiviral agent. PLoS ONE. 2015; 10:e122844.

14. Forns X, Lee SS, Valdes J, Lens S, Ghalib R, Aguilar H, et al. Glecaprevir plus pibrentasvir for chronic hepatitis $\mathrm{C}$ virus genotype $1,2,4,5$, or 6 infection in adults with compensated cirrhosis (EXPEDITION-1): a single-arm, open-label, multicentre phase 3 trial. Lancet Infect Dis. 2017;17:1062-8.

15. Pariser DM, Meinking TL, Bell M, Ryan WG. Topical $0.5 \%$ ivermectin lotion for treatment of head lice. N Engl J Med. 2012;367:1687-93.

16. Currie BJ, Harumal P, McKinnon M, Walton SF. First documentation of in vivo and in vitro ivermectin resistance in Sarcoptes scabiei. Clin Infect Dis. 2004;39:e8-12.

17. Caly L, Druce JD, Catton MG, Jans DA, Wagstaff KM. The FDA-approved drug ivermectin inhibits the replication of SARSCoV-2 in vitro. Antiviral Res. 2020;178:104787.

18. Kwo PY, Poordad F, Asatryan A, Wang S, Wyles DL, Hassanein $\mathrm{T}$, et al. Glecaprevir and pibrentasvir yield high response rates in patients with HCV genotype 1-6 without cirrhosis. J Hepatol. 2017;67:263-71.

19. Boonma $T$, Nutho $B$, Rungrotmongkol $T$, Nunthaboot $N$. Understanding of the drug resistance mechanism of hepatitis $\mathrm{C}$ 
virus NS3/4A to paritaprevir due to D168N/Y mutations: a molecular dynamics simulation perspective. Comput Biol Chem. 2019;83:107154.

20. Bagaglio S, Uberti-Foppa C, Morsica G. Resistance mechanisms in hepatitis $\mathrm{C}$ virus: implications for direct-acting antiviral use. Drugs. 2017;77:1043-55.

21. Senatore F, Jagadeesh G, Rose M, Pillai VC, Hariharan S, Liu Q, et al. FDA approval of angiotensin II for the treatment of hypotension in adults with distributive shock. Am J Cardiovasc Drugs. 2019;19:11-20.

22. Chayama K, Takahashi S, Toyota J, Karino Y, Ikeda K, Ishikawa $\mathrm{H}$, et al. Dual therapy with the nonstructural protein $5 \mathrm{~A}$ inhibitor, daclatasvir, and the nonstructural protein 3 protease inhibitor, asunaprevir, in hepatitis $\mathrm{C}$ virus genotype $1 \mathrm{~b}$-infected null responders. Hepatology. 2012;55:742-8.

23. Chua JV, Kottilil S. Sofosbuvir and velpatasvir: a stellar option for patients with decompensated hepatitis $\mathrm{C}$ virus (HCV) cirrhosis. Ann Transl Med. 2016;4:S8-S8.

24. Rose MJ, Merschman SA, Eisenhandler R, Woolf EJ, Yeh KC, Lin L, et al. High-throughput simultaneous determination of the HIV protease inhibitors indinavir and L-756423 in human plasma using semi-automated 96-well solid phase extraction and LC-MS/ MS. J Pharm Biomed Anal. 2000;24:291-305.

25. Carrion AF, Martin P. Safety and efficacy of elbasvir and grazoprevir for treatment of hepatitis C. Expert Opin Drug Saf. 2016;15:883-90.

26. Deeks ED. Ombitasvir/paritaprevir/ritonavir plus dasabuvir: a review in chronic HCV genotype 1 infection. Drugs. 2015;75:1027-38.

27. Gulick RM, Mellors JW, Havlir D, Eron JJ, Gonzalez C, McMahon D, et al. Treatment with indinavir, zidovudine, and lamivudine in adults with human immunodeficiency virus infection and prior antiretroviral therapy. $\mathrm{N}$ Engl J Med. 1997;337:734-9.

28. Brzoska T, Böhm M, Lügering A, Loser K, Luger TA. Terminal signal: Anti-inflammatory effects of $\alpha$-melanocyte- stimulating hormone related peptides beyond the pharmacophore. Adv Exp Med Biol. 2010;681:107-16.
29. Tong X, Le Pogam S, Li L, Haines K, Piso K, Baronas V, et al. In vivo emergence of a novel mutant $\mathrm{L} 159 \mathrm{~F} / \mathrm{L} 320 \mathrm{~F}$ in the NS5B polymerase confers low-level resistance to the hcv polymerase inhibitors mericitabine and sofosbuvir. $J$ Infect Dis. 2014;209:668-75.

30. Drożdżal S, Rosik J, Lechowicz K, Machaj F, Kotfis K, Ghavami $\mathrm{S}$, et al. FDA approved drugs with pharmacotherapeutic potential for SARS-CoV-2 (COVID-19) therapy. Drug Resist Updat. 2020;53:100719.

31. Ghosn J, Delaugerre C, Flandre P, Galimand J, Cohen-Codar I, Raffi F, et al. Polymorphism in gag gene cleavage sites of HIV-1 Non-B subtype and virological outcome of a first-line lopinavir/ ritonavir single drug regimen. PLoS ONE. 2011;6:e24798.

32. Harrison C. Coronavirus puts drug repurposing on the fast track. Nat Biotechnol. 2020;38:379-81.

33. Condra JH, Holder DJ, Schleif WA, Blahy OM, Danovich RM, Gabryelski LJ, et al. Genetic correlates of in vivo viral resistance to indinavir, a human immunodeficiency virus type 1 protease inhibitor. J Virol. 1996;70:8270-6.

34. Fischer J, Ganellin CR. Analogue-based drug discovery. Chem Int Newsmag IUPAC. 2014;32:12-5.

35. Croxtall JD, Perry CM. LopinavirRitonavir: a review of its use in the management of HIV-1 infection. Drugs. 2010;70:1885-915.

36. Ghosh AK, Bilcer G, Schiltz G. Syntheses of FDA approved HIV protease inhibitors. Synthesis (Stuttg). 2001;2001:2203-29.

37. Bonacker RC, Stoecklein KS, Locke JWC, Ketchum JN, Knickmeyer ER, Spinka CM, et al. Treatment with prostaglandin F2 $\alpha$ and an intravaginal progesterone insert promotes follicular maturity in advance of gonadotropin-releasing hormone among postpartum beef cows. Theriogenology. 2020;157:350-9.

38. Plummer M, de Martel C, Vignat J, Ferlay J, Bray F, Franceschi S. Global burden of cancers attributable to infections in 2012: a synthetic analysis. Lancet Glob Heal. 2016;4:e609-16.

Publisher's Note Springer Nature remains neutral with regard to jurisdictional claims in published maps and institutional affiliations. 